\title{
Feedback Inhibition Defines Transverse Processing Modules in the Lateral Amygdala
}

\author{
Rachel D. Samson, Éric C. Dumont, and Denis Paré \\ Center for Molecular and Behavioral Neuroscience, Rutgers State University, Newark, New Jersey 07102
}

The lateral amygdaloid (LA) nucleus is the main input station of the amygdala for sensory afferents. However, it is unclear how the lateral nucleus transforms these inputs, because its intrinsic connectivity is poorly understood. Here, we took advantage of the fact that glutamatergic neurons of the lateral nucleus send a primarily unidirectional projection to the basomedial nucleus. Consequently, it was possible to infer the targets of their intranuclear axons (projection cells vs inhibitory interneurons) by backfiring some projection neurons from the basomedial nucleus and analyzing evoked responses in other LA projection cells. Basomedial stimuli evoked markedly different synaptic responses depending on the orientation of the slices. In coronal slices (intact and decorticated), the prevalent response of LA neurons was an inhibition, regardless of the stimulation intensity. This inhibition was sensitive to $\mathrm{GABA}_{\mathrm{A}}$ and non-NMDA receptor antagonists, suggesting that it was mediated by the activation of GABAergic cells of the LA. In contrast, basomedial stimuli primarily evoked EPSPs in horizontal slices, regardless of the position of recorded neurons. In light of these findings, we conclude that the prevalent target of the intrinsic axon collaterals of projection cells depend on the rostrocaudal position of target neurons with respect to the parent cell body: inhibitory interneurons at rostrocaudal proximity versus other projection cells at a distance. Thus, feedback interneurons effectively divide the lateral nucleus in transverse processing modules that prevent runaway excitation within each module but allow intermixing of sensory information in the rostrocaudal plane.

Key words: amygdaloid complex; parvalbumin; interneurons; intrinsic connections; network; fear conditioning; emotion; guinea pig

\section{Introduction}

The distinguishing network properties supporting the involvement of the amygdala in fear expression (Davis, 2000; LeDoux, 2000) and memory consolidation (Cahill, 2000; McGaugh, 2000) remain elusive. It is clear, however, that the lateral amygdaloid (LA) nucleus plays a key role, because it receives most sensory inputs from the thalamus and cerebral cortex (Russchen, 1986; Turner and Herkenham, 1991; McDonald, 1998). However, the transformations performed by the LA on sensory afferents are unknown, because our understanding of its intrinsic circuit is rudimentary. The present study was undertaken to address this issue.

The LA nucleus contains two main cell types (for review, see McDonald, 1992a): (1) spiny multipolar projection cells with highly collateralized axons and (2) a heterogeneous class of aspiny (or sparsely spiny) local-circuit neurons. Projection cells account for the vast majority of LA neurons (McDonald, 1992b), they use glutamate (Smith and Paré, 1994) but not GABA (Carlsen, 1988; Paré and Smith, 1994) as a transmitter, and they contribute most, if not all, internuclear projections of the amygdala (Smith and Paré, 1994) (but see Stefanacci et al., 1992).

As in the cortex (for review, see Freund and Buzsáki, 1996; Kawaguchi and Kubota, 1997), local-circuit cells are morphologically and neurochemically heterogeneous. For example, subsets of GABAergic cells in the basolateral nucleus complex express

\footnotetext{
Received 0ct. 28, 2002; revised Dec. 17, 2002; accepted Dec. 17, 2002.

This work was supported by National Institute of Mental Health Grant MH-66856. We thank E. J. Lang for comments on a previous version of this manuscript.

Correspondence should be addressed to Denis Paré, Center for Molecular and Behavioral Neuroscience, Rutgers, The State University of New Jersey, 197 University Avenue, Newark, NJ 07102. E-mail: pare@axon.rutgers.edu. Copyright $\odot 2003$ Society for Neuroscience $\quad 0270-6474 / 03 / 231966-08 \$ 15.00 / 0$
}

somatostatin, neuropeptide $\mathrm{Y}$, cholecystokinin, or vasoactive intestinal peptide (McDonald and Pearson, 1989; Katona et al., 2001). In addition, calcium-binding proteins, such as parvalbumin (PV), also colocalize with GABA, but in a higher proportion of interneurons (Kemppainen and Pitkänen, 2000; McDonald and Betette, 2001).

As in the cortex, PV interneurons are believed to mediate feedback inhibition. Consistent with this view, projection cells form asymmetric (presumably excitatory) synapses on PV interneurons, whereas cortical axons do not (Smith et al., 2000). On the output side, PV cells form numerous inhibitory synapses on the soma, initial axonal segment, and proximal dendrites of projection cells (Smith et al., 1998). Under the light microscope, the density of these inhibitory terminals is such that they sometimes appear to delineate the soma and proximal processes of projection cells (Pitkänen and Amaral, 1993; Sorvari et al., 1995; Kemppainen and Pitkänen, 2000; McDonald and Betette, 2001).

The present study aimed to shed light on the spatial organization of feedback inhibition evoked in LA projection cells by backfiring their axons in the basomedial (BM) nucleus. Our results suggest that the prevalent target of the recurrent axon collaterals of projection cells vary with rostrocaudal distance between the parent cell and target neurons: inhibitory local-circuit cells in the same coronal plane and other projection cells at a distance.

\section{Materials and Methods}

Preparation of amygdala slices. Coronal (Fig. $1 \mathrm{~A}$ ) and horizontal (Fig. $1 \mathrm{~B}$ ) slices of the amygdala were obtained from Hartley guinea pigs (250-300 $\mathrm{gm})$. Experiments were done with the approval of the Rutgers University Institutional Research Board and in accordance with the NIH Guide to the Care and Use of Laboratory Animals. The animals were deeply anesthetized with pentobarbital (40 mg/kg, i.p.), ketamine (100 mg/kg, i.p.), 

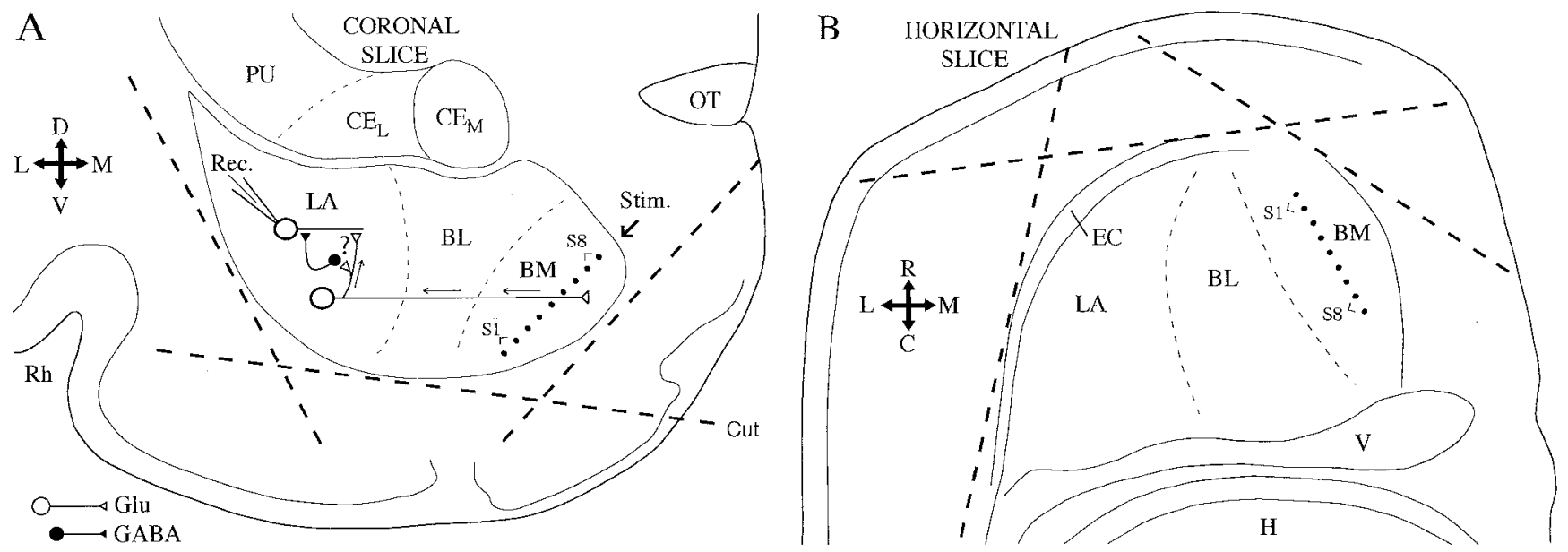

Figure 1. Scheme of coronal $(A)$ and horizontal $(B)$ amygdala slices. The orientation of the slices is indicated by the crosses on the left of each panel $(D$, dorsal; $V$, ventral; $L, l$ lateral; $M$, medial; $R$, rostral; $C$, caudal). Dashed lines indicate the position of knife cuts that were made in some experiments to remove cortical inputs. Dots in the BM nucleus represent the tip of stimulating (Stim.) electrodes. Whole-cell recordings (Rec.) of $L A$ projection neurons were performed. Their response to electrical stimuli applied in the BM nucleus were recorded. The circuit presumed to generate BM-evoked responses is indicated in $A . B L$, Basolateral nucleus; CEL, lateral sector of the central amygdaloid nucleus; CEM, medial sector of the central amygdaloid nucleus; EC, external capsule; $G l u$, glutamate; $H$, hippocampus; $P U$, putamen; $R h$, rhinal sulcus; $V$, ventricle.

and xylazine $(12 \mathrm{mg} / \mathrm{kg}$, i.p.) before decapitation. The brain was placed in an oxygenated solution $\left(4^{\circ} \mathrm{C}\right)$ containing the following (in $\mathrm{mM}$ ): 126 $\mathrm{NaCl}, 2.5 \mathrm{KCl}, 1.25 \mathrm{NaH}_{2} \mathrm{PO}_{4}, 1 \mathrm{MgCl}_{2}, 2 \mathrm{CaCl}_{2}, 26 \mathrm{NaHCO}_{3}$, and 10 glucose. Brain slices $(400 \mu \mathrm{m})$ were prepared using a vibrating microtome and stored for $1 \mathrm{hr}$ in an oxygenated chamber at $23^{\circ} \mathrm{C}$. One slice was then transferred to a recording chamber perfused with an oxygenated physiological solution $(2 \mathrm{ml} / \mathrm{min})$. The temperature of the chamber was gradually increased to $32^{\circ} \mathrm{C}$ before the recordings began.

Data recording and analysis. Whole-cell recordings were obtained with borosilicate pipettes filled with a solution containing the following (in $\mathrm{mm}$ ): $130 \mathrm{~K}$-gluconate, $10 \mathrm{HEPES}, 10 \mathrm{KCl}, 2 \mathrm{MgCl}_{2}, 2 \mathrm{ATP}-\mathrm{Mg}$, and 0.2 GTP-Tris. The $\mathrm{pH}$ was adjusted to 7.2 with $\mathrm{KOH}$ and osmolarity to $\sim 280$ mOsm. The liquid junction potential was $10 \mathrm{mV}$ with this solution, and the membrane potential $\left(V_{\mathrm{m}}\right)$ was corrected accordingly. The pipettes had a resistance of $4-8 \mathrm{M} \Omega$ when filled with the above solution. Recordings with series resistance $>15 \mathrm{M} \Omega$ were discarded. Current-clamp recordings were obtained with an Axoclamp 2B amplifier (Axon Instruments, Foster City, CA) under visual control using differential interference contrast and infrared video microscopy. Concentrations of drugs applied in the perfusate were as follows (in $\mu \mathrm{M}$ ): 10 bicuculline hydrochloride, 100 picrotoxin, and 20 6-cyano-7-nitroquinoxaline-2,3dione (CNQX).

An array of tungsten stimulating electrodes $(80 \mu \mathrm{m}$ in diameter; 80 $\mathrm{k} \Omega$ ) was positioned in the BM nucleus as shown in Figure 1 (dots). Electrical stimuli consisted of $100 \mu$ sec current pulses $(0.1-1 \mathrm{~mA})$ passed through neighboring electrodes. Synaptic responses were elicited from a $V_{\mathrm{m}}$ of approximately $-65 \mathrm{mV}$ as determined by intracellular current injection. When studying synaptic responses elicited by electrical stimuli, the stimulation intensity at the $\mathrm{BM}$ site closest to the recorded cell was increased gradually in steps of $50 \mu \mathrm{A}$ until a response was evoked. Subsequently, all of the stimulation sites were scanned sequentially at 1.5 times the threshold intensity (usually between 0.15 and $0.35 \mathrm{~mA}$ ). Three or more stimuli were applied at each site and averaged independently. A site was considered effective only if, at a particular stimulus intensity, at least three of the four stimuli elicited a response at a constant latency. Synaptic events with amplitudes $<0.5 \mathrm{mV}$ were ignored. For statistical analyses, the BM stimuli eliciting the largest responses was determined for each cell, treating EPSPs and IPSPs separately. Only these average values are reported below. Values are expressed as mean \pm SEM. To determine whether response amplitudes between cell groups differed significantly, $t$ tests were computed using a fixed level of significance $(p<0.05)$.

Analyses were performed off-line with the software IGOR (WaveMetrics, Lake Oswego, OR) and homemade software running on Macintosh microcomputers (Apple Computers, Cupertino, CA). The input resistance $\left(R_{\text {in }}\right)$ of the cells was estimated in the linear portion of currentvoltage plots.

\section{Results}

\section{Database}

Previous Golgi studies have revealed that spiny multipolar projection cells account for the vast majority of neurons in the LA nucleus $(\sim 85 \%)$ compared with the generally smaller-diameter aspiny interneurons (for review, see McDonald, 1992b). Thus, random samples of LA cells should be composed primarily of projection neurons. Nevertheless, we attempted to further restrict our attention to projection cells (1) by aiming our electrodes toward large-diameter somatic profiles and (2) by considering only neurons displaying electroresponsive features that are characteristic of projection cells. These included a lack of spontaneous discharges at rest, generation of 4-10 Hz oscillations on membrane depolarization beyond $-60 \mathrm{mV}$, and generation of spike trains exhibiting frequency accommodation with additional depolarization (Washburn and Moises, 1992a; Rainnie et al., 1993; Paré et al., 1995b; Lang and Paré, 1998; Pape and Driesang 1998; Faber et al., 2001).

Using these criteria, a total of 185 LA neurons with a $V_{\mathrm{m}}$ negative to $-60 \mathrm{mV}$ and generating overshooting action potentials were recorded (96 and 89 in coronal and horizontal slices, respectively). Overall, 35\% of these cells (or 65 neurons) were responsive to BM stimuli (32 and 33 in coronal and horizontal slices, respectively). Twenty-three of these cells were recorded in decorticated slices (Fig. 1, dashed lines). On average, BMresponsive neurons had a resting potential of $-79 \pm 1 \mathrm{mV}$ and an input resistance of $263 \pm 19 \mathrm{M} \Omega$ and generated action potentials of $76 \pm 1 \mathrm{mV}$ lasting $1.50 \pm 0.05 \mathrm{msec}$ at half-amplitude. To measure action potential amplitudes, a series of depolarizing current pulses of increasing amplitude (in steps of $0.01 \mathrm{nA}$ ) was applied from rest. We measured the amplitude of the first spike that was elicited by calculating the difference between the threshold and peak voltages. The rest of this study will focus on this subset of BM-responsive LA neurons.

Note that the low proportion of responsive neurons does not result from the scarcity of projections from the LA to the BM 
nucleus but rather from the fact that we searched the entire LA nucleus for responsive cells and found them to be concentrated in the core of the LA nucleus (Krettek and Price, 1978). However, we did not record cells in the most medial part of the LA, to which the BM sends a minor projection (Paré et al., 1995a; Savander et al., 1997).

\section{BM-evoked responses in coronal slices}

Because we aimed to identify the targets of the intranuclear collaterals of LA projection cells by backfiring some of them from the BM nucleus (Fig. 1A), we first examined whether BM stimuli could evoke antidromic spikes in LA neurons at rest. Antidromic spikes were identified as such when they arose directly from the baseline (Fig. $2 A$ ), occurred at a fixed latency (Fig. $2 A$ ), and collided with spikes elicited by depolarizing current pulses within twice the antidromic response latencies (Fig. 2B1) but not with longer intervals (Fig. 2B2).

Using these criteria, it was determined that $22 \%$ of LA neurons (or 7 of 32 cells) were antidromically invaded from one or more BM site(s) (average, $1.9 \pm 0.3$ sites; latency, $6.73 \pm 0.98$ $\mathrm{msec})$, but no orthodromic spikes were observed. When cells were backfired from more than one BM stimulation site (four of seven cells), the effective sites were not necessarily contiguous (average interval between effective sites, $2.3 \pm 0.9$ ), suggesting that our stimulation method was relatively selective.

When depolarized to approximately $-65 \mathrm{mV}$, the prevalent response of LA neurons to BM stimuli was an inhibition in coronal slices. A representative example of this is shown in Figure $3 A$. Note that, when BM stimuli evoked EPSPs, they had a low amplitude (average, $1.8 \pm 0.4 \mathrm{mV}$ compared with $4.3 \pm 0.6 \mathrm{mV}$ for the IPSPs). In fact, as many as $64 \%$ of responsive neurons (or 20 of 32 cells) displayed maximal BM-evoked EPSPs $\leq 1 \mathrm{mV}$.

Importantly, the character of BM-evoked responses did not change with the stimulation intensity $(n=11)$. This is shown in Figure $3 B$, illustrating the effects of BM stimuli of gradually increasing intensity (numbers on the right). In this representative
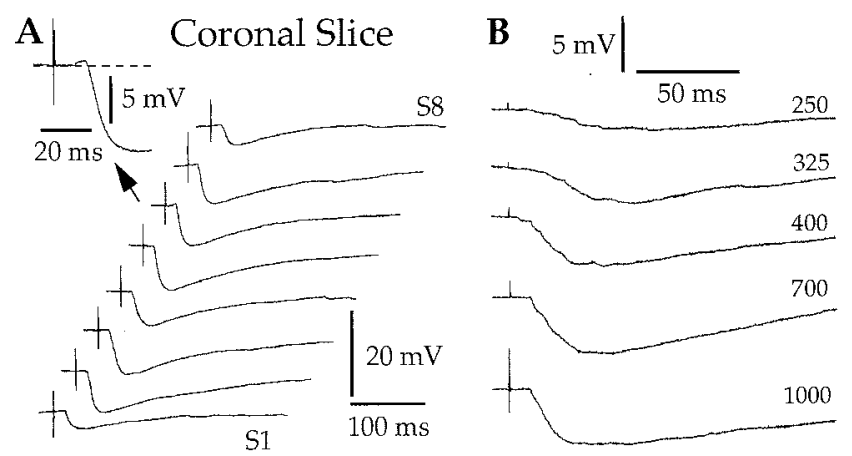

Figure 3. In coronal slices, the character of BM-evoked responses does not vary with the exact stimulation site $(A)$ or the stimulation intensity $(B) . A$, Each sweep represents the average of three responses to stimuli applied at eight different dorsoventral levels of the BM nucleus (S1-S8) (Fig. 1A, dots). The inset in $A$ shows the response with the largest excitatory component seen in this neuron. $B$, Changing the intensity of BM stimuli (numbers to the right; in microamperes) does not modify the character of evoked responses. Two different $L A$ neurons depolarized to $-65 \mathrm{mV}$ by steady injection of $0.03(A)$ and $0.08 \mathrm{nA}(B)$. Rest was -75 and $-87 \mathrm{mV}$, respectively. neuron, apparently pure inhibitory responses were evoked from threshold intensity to the strongest stimuli our equipment could deliver. However, for consistency, all response amplitudes reported in this study were obtained with BM stimuli equal to 1.5 times the threshold intensity (see Materials and Methods).

In most cells ( $71 \%$, or 23 of 32 cells), the BM-evoked inhibition was monophasic (Fig. $4 A$ ), lasted $261 \pm 23 \mathrm{msec}$, reversed at $-78 \pm 2.2 \mathrm{mV}$, and was completely abolished by bicuculline hydrochloride or picrotoxin $(n=6)$ (Fig. $4 C)$, suggesting a mediation by $\mathrm{GABA}_{\mathrm{A}}$ receptors (Rainnie et al., 1991; Washburn and Moises, 1992b; Danober and Pape, 1998; Martina et al., 2001a). In a few cells, however ( $29 \%$, or 9 of 32 cells), BM stimuli evoked longer ( $552 \pm 28$ msec) biphasic IPSPs (Fig. 4B), with the late phase reversing at a significantly more negative $V_{\mathrm{m}}(-89.2 \pm 1.6 \mathrm{mV})$ than the early one $(-76.5 \pm 0.7 \mathrm{mV}$; paired $t$ test, $p<0.05)$. The relatively negative reversal potential of the late IPSP coupled to its resistance to $\mathrm{GABA}_{\mathrm{A}}$ antagonists $(n=3)$ (Fig. $4 D)$ suggests that it is mediated by $\mathrm{GABA}_{\mathrm{B}}$ receptors (see references cited above). It should be noted that all antidromically responsive LA neurons also displayed IPSPs in response to BM stimuli.

To determine whether these IPSPs were generated by BM neurons activated directly by the electrical stimuli versus distant neurons recruited synaptically, we tested the effect of the nonNMDA antagonist CNQX on BM-evoked responses. Addition of CNQX to the perfusate abolished BM-evoked EPSPs and IPSPs $(n=4)$ (Fig. $5 A)$. This indicates that the IPSPs were not generated by GABAergic cells of the BM nucleus projecting to the LA but rather resulted from the glutamatergic activation of GABAergic cells located at a distance from the stimulation electrodes, possibly in the LA or adjacent cortical fields.

To test the latter possibility, we studied the effect of BM stimuli on LA neurons $(n=12)$ recorded in decorticated slices (Fig. $1 A$, dashed lines). As shown in Figure 5B, cortical cuts (Fig. $1 A$, dashed lines) did not modify BM-evoked response patterns. To test this quantitatively, we compared the average amplitude of BM-evoked EPSPs and IPSPs in intact versus decorticated coronal slices (EPSPs, $1.27 \pm 0.27$ vs $0.83 \pm 0.36 \mathrm{mV}$; IPSPs, $-4.97 \pm$ $0.65 \mathrm{vs}-6.1 \pm 1.3 \mathrm{mV})$. Differences in response amplitude did not reach statistical significance ( $t$ test, $p>0.05$ ). Moreover, the number of BM stimulation sites evoking IPSPs did not differ in the two groups of cells. By exclusion, these results suggest that the GABAergic neurons generating BM-evoked IPSPs are located in the LA and that the cortex is not required to activate them. 


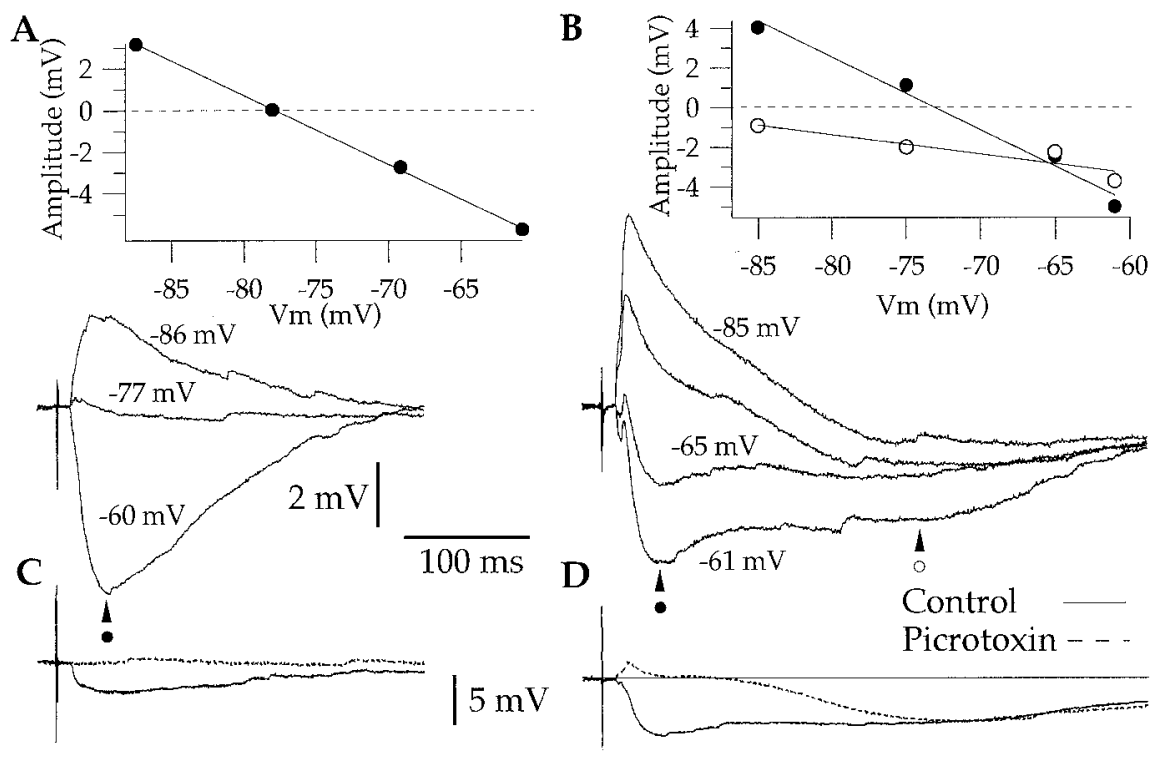

Figure 4. Monophasic $(A)$ and biphasic $(B)$ BM-evoked IPSPs in coronal slices. Each sweep is the average of three responses Both panels show BM-evoked responses at different membrane potentials, as determined by steady intracellular current injection. Effect of picrotoxin on monophasic ( $C$ and biphasic (D) BM-evoked IPSPS. C, D, Continuous lines indicate control responses, whereas dashed lines indicate responses observed in the presence of picrotoxin. Four different LA neurons with resting potentials of $-87,-82,-86$, and $-82 \mathrm{mV}$, respectively.

However, if we assume that BM-evoked responses resulted from the antidromic invasion of LA axons contacting other LA neurons, the prevalence of inhibitory responses to BM stimuli is surprising. Indeed, previous ultrastructural studies have revealed that the axons of LA projection cells are enriched in glutamate and form only asymmetrical synapses, usually (88\%) with dendritic spines in the LA (Smith and Paré, 1994). Moreover, similar findings were obtained in other nuclei of the basolateral complex (Paré et al., 1995a). Another possibility, namely that BM-evoked responses resulted from activation of the weak BM projection to the most medial sector of the LA nucleus, is also inconsistent with our observations, because BM axons also form excitatory synapses, usually with other LA projection cells (Paré et al., 1995a).

This led us to consider the possibility that the strategic location of inhibitory terminals in the perisomatic region of LA projection cells (Smith et al., 1998) was shunting excitatory inputs ending in more distal dendritic segments. To test this, we examined the response profiles of six projection cells before versus after administration of the $\mathrm{GABA}_{\mathrm{A}}$ antagonists bicuculline or picrotoxin. As shown in Figure $5 C$, GABA $_{\mathrm{A}}$ blockade did not uncover large excitatory responses but produced a minor yet statistically significant increase in EPSP amplitude (paired $t$ test, $p<0.05 ; 1.74 \pm 0.74$ vs $2.81 \pm 0.77$ $\mathrm{mV}$ in the absence and presence of picrotoxin, respectively). In one cell, however (Fig. 5C), picrotoxin increased the number of BM sites eliciting antidromic spikes and/or reduced the antidromic response latency.

This suggests that cell-to-cell variations in the conduction velocity of LA axons are such that the feedback inhibition produced by the activation of fasterconducting LA axons can block the antidromic responses of LA neurons with lower conduction velocities. In addition, this implies that some of the BM-evoked depolarizations seen in control conditions are not EPSPs but rather the somatic reflection of axonal (" $\mathrm{m}$ ”) spikes that propagated retrogradely to the soma but were prevented from evoking full action potentials by feedback inhibition.

\section{BM-evoked responses in horizontal slices}

At odds with ultrastructural findings, the above-described results suggested that coronal slices contain few connected BMprojecting LA neurons but preserve the links between projection cells and feedback interneurons. This discrepancy is particularly striking because BM-evoked EPSPs should be monosynaptic, whereas IPSPs are disynaptic. However, it remained possible that the low amplitude of BM-evoked EPSPs resulted simply from electrotonic attenuation. We reasoned that this possibility could be ruled out if, in horizontal slices, BM-evoked responses were composed primarily of EPSPs, as predicted by ultrastructural observations.
A

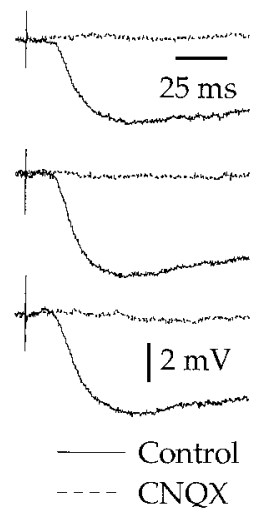

B Decorticated Coronal Slice

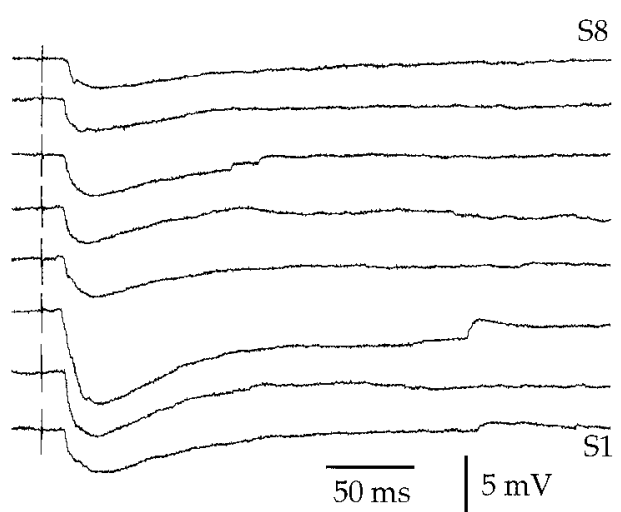

C

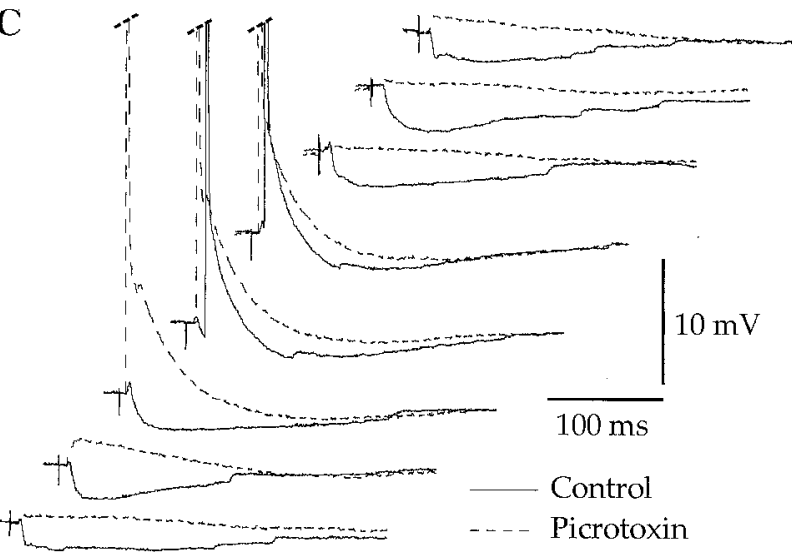

Figure 5. BM-evoked IPSPs are abolished by CNQX and GABA receptor antagonists but persist in decorticated slices. A, BM-evoked IPSPs recorded before (Control, continuous lines) and after (dashed lines) addition of CNQX to the perfusate. B, BM-evoked IPSPs recorded in a decorticated slice. Each sweep represents the average of three responses to stimuli applied at eight different BM stimulation sites (S1-S8) (Fig. 1). C, BM-evoked responses recorded before (Control, continuous line) and after (dashed lines) addition of picrotoxin to the perfusate. Three different LA neurons depolarized to $-65 \mathrm{mV}$ by steady injection of $0.03(A), 0.07(B)$, and $0.08 \mathrm{nA}(C)$. Rest was $-76,-84$, and $-86 \mathrm{mV}$, respectively. 

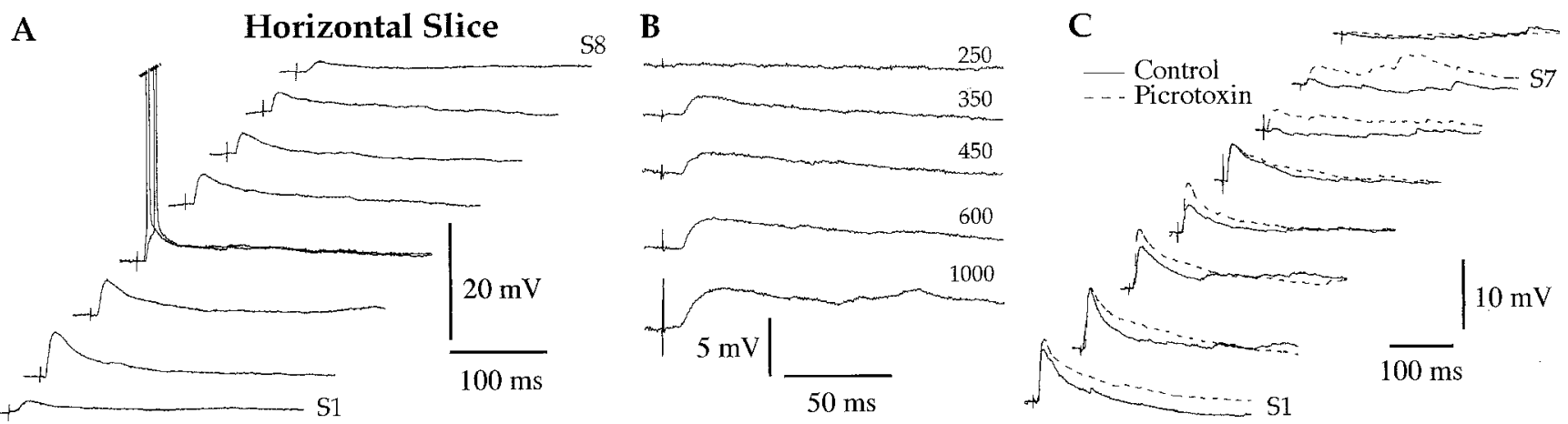

Figure 6. In horizontal slices, the prevalent response of $L A$ neurons to BM stimuli is an excitation. $A$, Response profile of an $L A$ neuron to electrical stimuli applied at various rostrocaudal levels of the BM nucleus (S1-S8) (Fig. 1B, dots). B, Changing the intensity of BM stimuli (numbers to right; in microamperes) does not modify the character of evoked responses. Note the long-latency response components evoked at the highest stimulation intensity. C, BM-evoked responses recorded before (Control, continuous line) and after (dashed lines) addition of picrotoxin to the perfusate. The action potentials seen before and/or after picrotoxin were antidromic spikes. Three different LA neurons depolarized to $-65 \mathrm{mV}$ by steady injection of $0.01(A), 0.03(B)$, and $0.09 \mathrm{nA}(C)$. Rest was $-68,-75$, and $-85 \mathrm{mV}$, respectively.

To test this idea, we studied the response of presumed projection neurons to BM stimuli in horizontal slices. In this condition, $21 \%$ of LA neurons (or 7 of 33 cells) were antidromically invaded from one or more BM stimuli (average, $2.2 \pm 0.3$ sites; latency, $6.66 \pm 0.82 \mathrm{msec}$ ), and $9 \%$ of cells were orthodromically responsive. Note that the proportion of antidromically responsive cells in horizontal versus coronal sections is virtually identical ( $\chi^{2}$ test, $p>0.05)$.

At odds with the hypothesis that the low amplitude of EPSPs in coronal slices is caused by electrotonic attenuation, the prevalent BM-evoked response seen in horizontal slices was an excitation (Fig. 6A). In fact, as many as $46 \%$ of responsive neurons displayed IPSPs $\leq 1 \mathrm{mV}$ compared with $6 \%$ in coronal slices. Importantly, the character of BM-evoked response was not a function of the stimulation intensity. To test this, we applied BM stimuli of gradually increasing intensity in cells showing apparently pure excitatory responses $(n=10)$. As shown in the representative neuron of Figure $6 B$, apparently pure excitatory responses were evoked from threshold intensity to the strongest stimuli our equipment could deliver. All antidromically responsive cells also displayed this response profile.

Moreover, the same response profile was seen in horizontal slices with cortical cuts $(n=11$; data not shown). To test this quantitatively, we compared the average amplitude of BMevoked EPSPs and IPSPs in intact versus decorticated horizontal slices (EPSPs, $3.41 \pm 0.44$ vs $5.01 \pm 1.10 \mathrm{mV}$; IPSPs, $1.80 \pm 0.35$ vs $1.12 \pm 0.21 \mathrm{mV}$ ). Differences in response amplitude did not reach statistical significance ( $t$ test, $p>0.05$ ). Figure $7 A$ compares the frequency distribution of EPSP and IPSP amplitudes in LA neurons recorded in coronal (thick lines; $n=32$ ) and horizontal (thin lines; $n=33$ ) slices. Despite some overlap in these amplitude distributions, EPSP amplitudes tended to be higher in horizontal slices (Fig. 7A1), whereas IPSPs predominated in coronal slices (Fig. 7A2). Both differences in average response amplitudes (see figure legend) were significant (unpaired $t$ tests, $p<0.05$ ).

Importantly, the contrasting response profiles seen in horizontal versus coronal slices were not dependent on the rostrocaudal position of recorded cells. This is shown in Figure $7 B$, which plots maximal BM-evoked EPSP ( $y$-axis, positive values) and IPSP ( $y$-axis, negative values) amplitudes as a function of rostrocaudal position (x-axis) in coronal (Fig. 7B1) and horizontal (Fig. 7B2) slices.

Although there was generally little overt inhibition in horizontal slices, $\mathrm{GABA}_{\mathrm{A}}$ blockade with picrotoxin or bicuculline
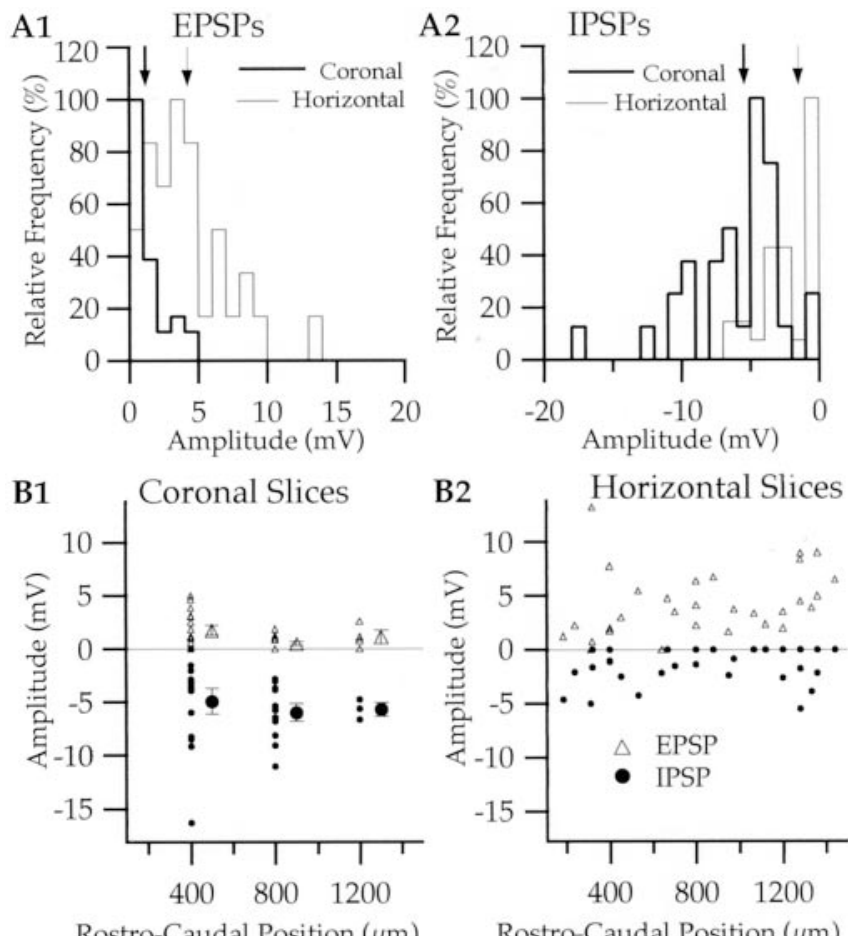

Rostro-Caudal Position $(\mu \mathrm{m})$

Figure 7. Comparison between BM-evoked EPSP and IPSP amplitudes in coronal and horizontal slices. A, Normalized frequency distribution of EPSP (A1) and IPSP (A2) amplitudes as recorded in coronal (thick lines; $n=32$ ) and horizontal (thin lines; $n=33$ ) slices. In both cases, the frequency distribution was normalized so that the mode of each histogram was equal to $100 \%$. The thick and thin arrows (top of histograms) mark the average amplitude of evoked responses in coronal and horizontal slices, respectively. $B$, Amplitude of BM-evoked responses ( $y$-axis) as a function of rostrocaudal level ( $x$-axis) in coronal (B1) and horizontal (B2) slices. In horizontal sections (B2), rostrocaudal position was defined with respect to the anterior limb of the external capsule (Fig. $1 B$ ), whereas the serial position of the slice, from rostral to caudal, was used in coronal slices (B1). In coronal slices, EPSPs $>0.5 \mathrm{mV}$ were observed in 19 of 32 neurons. In horizontal slices, IPSPs $>0.5$ $\mathrm{mV}$ were recorded in 17 of 33 neurons. The larger symbols in $B 1$ indicate average response amplitudes. The numbers of cells recorded in the first, second, and third slices are 16, 12, and 4. Some data points cannot be seen because they mask each other. Note that a smaller rostrocaudal extent of the LA was sampled in coronal slices because the medial limit of the LA proved difficult to define at the rostral pole of the amygdala. No difference was found between the input resistance of neurons recorded in coronal $(252 \pm 22 \mathrm{M} \Omega)$ and horizontal $(269 \pm 36 \mathrm{M} \Omega)$ slices; unpaired $t$ test, $p>0.05$. 
$(n=7)$ (Fig. 6C) did produce significant enhancement of EPSP amplitudes $(3.57 \pm 0.32$ in control conditions compared with $5.39 \pm 0.67 \mathrm{mV}$ after bicuculline or picrotoxin application; $t$ test, $p<0.05)$.

However, application of $\mathrm{GABA}_{\mathrm{A}}$ antagonists in a population of interconnected glutamatergic neurons not only blocks inhibition but also increases the number of neurons excited in a suprathreshold manner by BM stimuli. Thus, the increase in EPSP amplitudes does not reflect the direct impact of IPSPs on recorded cells. As a result, the only way to assess the importance of inhibition in horizontal versus coronal slices is to affect inhibitory responses by manipulations of the intracellular milieu that will not modify the excitatory drive to LA cells.

To this end, 10 additional BM-responsive LA neurons were recorded in horizontal $(n=5)$ and coronal $(n=5)$ slices using a pipette solution containing a high chloride concentration, thus reversing the transmembrane chloride gradient. This was achieved by replacing K-gluconate with an equimolar amount of $\mathrm{KCl}$. As in previous experiments, these tests were performed at a membrane potential of $-65 \mathrm{mV}$, after correction of the junction potential. Subsequently, we tested whether the peak absolute amplitude of BM-evoked response differed with a low versus high intracellular chloride concentration. In horizontal slices, the different chloride concentrations had a statistically insignificant effect on the amplitude of peak BM-evoked responses (control, $4.1 \pm 0.6 \mathrm{mV}$; high chloride, $5.6 \pm 1.1 \mathrm{mV}$; unpaired $t$ test, $p>$ 0.05 ), whereas a large difference was seen in coronal slices (control, $-4.9 \pm 0.7 \mathrm{mV}$; high chloride, $6.5 \pm 1.1 \mathrm{mV}$; unpaired $t$ test, $p<0.05)$.

\section{Discussion}

This study was undertaken to characterize the intrinsic circuit of the LA nucleus. Taking advantage of the primarily unidirectional nature of LA-to-BM projections, we aimed to infer the target of the recurrent axon collaterals of projection cells. To this end, we analyzed the responses evoked in LA projection neurons when some of them were backfired from the BM nucleus. Although the probability of backfiring projection cells was similar in horizontal and coronal slices, the character of BM-evoked responses varied depending on the slice orientation. In coronal slices, the prevalent response evoked by BM stimuli was an inhibition. This inhibition was also present after decortication but was sensitive to CNQX, suggesting that it was generated by GABAergic LA neurons. In contrast, the prevalent BM-evoked response in horizontal slices was an excitation that persisted after decortication. In the following account, we consider the origin of these differences and propose a new model of the intrinsic LA circuit.

\section{The intrinsic circuit of the LA nucleus is spatially heterogeneous}

Before this study, the sole source of data on the intranuclear targets of LA projection cells was electron microscopic (EM) observations of anterogradely labeled axons in the LA nucleus. It was found that axon terminals were enriched in glutamate but not GABA and that they formed only asymmetrical synapses, typically ( $88 \%$ of terminals) with dendritic spines (Smith and Paré, 1994). Because the dendrites of local-circuit cells are aspiny or sparsely spiny (McDonald, 1992a), these results suggested that the prevalent target of LA projection cells were other projection neurons.

In light of these findings, we expected BM stimuli to evoke large excitatory responses but observed the opposite in coronal slices. The possibility that proximal inhibitory inputs prevented us from observing distal EPSPs seems unlikely, because $\mathrm{GABA}_{\mathrm{A}}$ blockade produced only minor increases $(\sim 1 \mathrm{mV})$ in the excitatory component of $\mathrm{BM}$-evoked responses. Moreover, even in the presence of $\mathrm{GABA}_{\mathrm{A}}$ antagonists, BM-evoked EPSPs in coronal slices remained significantly lower in amplitude than those seen in horizontal slices bathed in control media. In contrast, IPSP amplitudes were higher in coronal than horizontal slices. The incontrovertible implication of these observations is that the connectivity of projection cells and/or that of feedback interneurons is heterogeneous in space.

\section{The prevalent targets of LA projection cells vary with rostrocaudal distance to target}

To infer the source(s) of this heterogeneity, we first consider BM-evoked EPSPs, because they involve only projection cells. From this standpoint, there are two possibilities: either (1) the dendritic tree of projection cells is not equally extensive in different planes or (2) their intrinsic axon collaterals are more or less likely to contact other projection cells, depending on their position. According to the first scenario, the low amplitude of BMevoked EPSPs in coronal slices would imply that the dendrites of projection cells do not branch in the coronal plane at the level of the soma. However, this possibility is not supported by the numerous studies on the morphological properties of projection cells (Washburn and Moises, 1992a; Rainnie et al., 1993; Paré et al., 1995b; Lang and Paré, 1998; Faber et al., 2001); the dendrites of projection cells branch extensively in the coronal plane. By exclusion, we conclude that BM-projecting neurons form fewer synapses with projection cells located in their immediate vicinity compared with those located at some distance in the rostrocaudal axis.

Nevertheless, the presence of BM-evoked IPSPs in coronal slices indicates that the axon collaterals of projection cells do arborize in the rostrocaudal proximity of their soma. This suggests that the prevalent targets of the intrinsic axon collaterals of projection cells vary with rostrocaudal distance to the target. Thus, our study implies that processing of sensory inputs in the LA is constrained by a network of intrinsic connections characterized by a hitherto unsuspected degree of spatial specificity. Interestingly, a similar pattern of organization was found in the perirhinal cortex, a cortical field connected reciprocally to the LA nucleus (Biella et al., 2001; Martina et al., 2001b).

We now turn to the origin of the differences in the amplitude of BM-evoked IPSPs as a function of the slice orientation. The above-described considerations indicate that the axons of projection cells collateralize extensively in the rostrocaudal axis. Thus, in principle, feedback interneurons have the opportunity to receive inputs from projection cells in coronal and horizontal slices, yet IPSPs generally had a low amplitude in horizontal slices. Because projection cells contact inhibitory neurons in their immediate rostrocaudal vicinity, one would expect at least some BM stimuli to evoke large IPSPs in all cells recorded in horizontal slices.

There are a number of possible interpretations for these observations. One possibility is that feedback interneurons and the projection cells they inhibit are not located in the same dorsoventral plane. This would minimize feedback inhibition in $400-\mu \mathrm{m}$ thick horizontal slices. A second and more parsimonious possibility takes into account the fact that feedback interneurons receive much excitation from projection cells located at the same rostrocaudal level (see above). In coronal slices, however, BM stimuli backfired LA neurons distributed over $\sim 1.5 \mathrm{~mm}$ in the dorsoventral plane. This implies that horizontal slices reduced the number of functional connections between projection cells 
and feedback interneurons, thus accounting for the paucity of inhibition.

An important task for future experiments will be to test these various hypotheses by reconstructing the axonal and dendritic arbors of physiologically identified feedback $(\mathrm{PV}+)$ interneurons and projection cells. If their targets are identified at the EM level, it will be possible to determine whether projection cells contact fewer feedback interneurons as the rostrocaudal distance from the parent soma increases, an issue that could not be addressed in this study.

\section{Could BM-to-LA projections account for differences seen depending on the slice orientation?}

Tracing studies have revealed a small projection from the BM to LA (Paré et al., 1995a; Savander et al., 1997). This projection is dense but targets only a thin crescent of the LA in its most medial margin. EM observations have revealed that BM axon terminals form exclusively asymmetric synapses, usually with dendritic spines in the LA (Paré et al. 1995a). Although the possibility that this projection was recruited by BM stimuli cannot be ruled out, it should be noted that we did not record from the most medial margin of the LA, in which this projection ends. Moreover, assuming that our responses were mediated by this pathway, a spatially heterogeneous connectivity would still be needed to account for the difference between coronal and horizontal slices, unless the BM-to-LA projection was differentially preserved, depending on the slice orientation. Although this possibility cannot be ruled out, it is inconsistent with the anatomic data. Indeed, the trajectory of LA-projecting BM axons suggests that they would be preserved in coronal and horizontal slices (Paré et al., 1995a).

\section{Implications for information processing in the LA nucleus}

Golgi studies have emphasized the spatially limited axonal arbor of LA interneurons compared with the extensive, highly collateralized axonal arbors of projection cells (McDonald, 1992a). In light of these observations, the finding that projection cells prevalently contact different cell types, depending on rostrocaudal distance to target, has important implications. Indeed, it suggests that feedback interneurons effectively divide the core of the LA nucleus in transverse processing modules whose dimensions are set by (1) the rostrocaudal extent of feedback inhibitory axons and (2) the distance at which the prevalent target of projection cells shifts from feedback interneurons to projection cells.

According to this view, suprathreshold activation of a circumscribed group of projection cells by sensory inputs would evoke feedback inhibition at the same transverse level and a wave of excitation at a distance. Given that thalamic and cortical afferents conveying sensory inputs of different modalities form a complex mosaic of termination zones in the LA (Russchen, 1986; Turner and Herkenham, 1991; McDonald, 1998), this model would allow intermixing of sensory information in the rostrocaudal axis while preventing runaway excitation by means of a rostrocaudally limited feedback inhibitory circuit.

This model is consistent with previous in vivo observations of inhibition in the LA nucleus (Lang and Paré, 1997a), in which it was found that low-intensity electrical stimuli delivered at various rostrocaudal levels of the perirhinal cortex elicited EPSPs. As the stimulation intensity was increased, the character of the response shifted toward inhibition until cortically evoked EPSPs were almost completely superseded. Although a synaptically activated $\mathrm{Ca}^{2+}$-dependent $\mathrm{K}^{+}$conductance contributed to the attenuation of EPSPs (Lang and Paré, 1997b; Danober and Pape, 1998), these results suggested the existence of a threshold in the number of activated projection cells above which inhibition took over. In light of the present study, we hypothesize that feedback inhibitory circuits recruited by (and acting on) a transversely circumscribed group of projection cells contributed to this behavior.

Although the identity of feedback interneurons remains to be established, the quasi-exclusive innervation of PV interneurons by intrinsic inputs (Smith et al., 2000) suggests that they represent likely candidates. However, the contribution of feedforward interneurons that receive convergent inputs from various sources (Szinyei et al., 2000) should not be overlooked.

\section{References}

Biella G, Uva L, de Curtis M (2001) Network activity evoked by neocortical stimulation in area 36 of the guinea pig perirhinal cortex. J Neurophysiol $86: 164-172$.

Cahill L (2000) Modulation of long-term memory storage in humans by emotional arousal: adrenergic activation and the amygdala. In: The amygdala: a functional analysis (Aggleton JP, ed), pp 425-445. Oxford: Oxford University Press.

Carlsen J (1988) Immunocytochemical localization of glutamate decarboxylase in the rat basolateral amygdaloid nucleus, with special reference to GABAergic innervation of amygdalostriatal projection neurons. J Comp Neurol 273:513-526.

Danober L, Pape HC (1998) Mechanisms and functional significance of a slow inhibitory potential in neurons of the lateral amygdala. Eur J Neurosci 10:853-867.

Davis M (2000) The role of the amygdala in conditioned and unconditioned fear and anxiety. In: The amygdala: a functional analysis (Aggleton JP, ed), pp 213-287. Oxford: Oxford University Press.

Faber E, Callister RJ, Sah P (2001) Morphological and electrophysiological properties of principal neurons in the rat lateral amygdala in vitro. J Neurophysiol 85:714-723.

Freund TF, Buzsáki G (1996) Interneurons of the hippocampus. Hippocampus 6:347-470.

Katona I, Rancz EA, Acsády L, Ledent C, Mackie K, Hájos N, Freund TF (2001) Distribution of CB1 cannabinoid receptors in the amygdala and their role in the control of GABAergic transmission. J Neurosci 21:9506-9518.

Kawaguchi Y, Kubota Y (1997) GABAergic cell subtypes and their synaptic connections in rat frontal cortex. Cereb Cortex 7:476-486.

Kemppainen S, Pitkänen A (2000) Distribution of parvalbumin, calretinin, and calbindin-D28k immunoreactivity in the rat amygdaloid complex and colocalization with gamma-aminobutyric acid. J Comp Neurol 426:441-467.

Krettek JE, Price JL (1978) A description of the amygdaloid complex in the rat and cat with observations on intra-amygdaloid axonal connections. J Comp Neurol 178:255-280.

Lang EJ, Paré D (1997a) Similar inhibitory processes dominate the responses of cat lateral amygdaloid projection neurons to their various afferents. J Neurophysiol 77:341-352.

Lang EJ, Paré D (1997b) Synaptic and synaptically activated intrinsic conductances underlie inhibitory potentials in cat lateral amygdaloid projection neurons in vivo. J Neurophysiol 77:353-363.

Lang EJ, Paré D (1998) Synaptic responsiveness of interneurons of the cat lateral amygdaloid nucleus. Neuroscience 83:877-889.

LeDoux JE (2000) Emotion circuits in the brain. Annu Rev Neurosci 23:155-184.

Martina S, Royer S, Paré D (2001a) Cell type specific GABA responses and chloride homeostasis in the cortex and amygdala. J Neurophysiol 86:2887-2895.

Martina M, Royer S, Paré D (2001b) Propagation of neocortical inputs in the perirhinal cortex. J Neurosci 21:2878-2888.

McDonald AJ (1992a) Cell types and intrinsic connections of the amygdala. In: The amygdala: neurobiological aspects of emotion, memory, and mental dysfunction (Aggleton JP, ed), pp 69-76. New York: Wiley-Liss.

McDonald AJ (1992b) Projection neurons of the basolateral amygdala: a correlative Golgi and retrograde tract tracing study. Brain Res Bull 28:179-185.

McDonald AJ (1998) Cortical pathways to the mammalian amygdala. Prog Neurobiol 55:257-332. 
McDonald AJ, Betette RL (2001) Parvalbumin-containing neurons in the rat basolateral amygdala: morphology and co-localization of calbindinD(28k). Neuroscience 102:413-425.

McDonald AJ, Pearson JC (1989) Coexistence of GABA and peptide immunoreactivity in non-pyramidal neurons of the basolateral amygdala. Neurosci Lett 100:53-58.

McGaugh JL (2000) Memory: a century of consolidation. Science 287:248-251.

Pape HC, Driesang RB (1998) Ionic mechanisms of intrinsic oscillations in neurons of the basolateral amygdaloid complex. J Neurophysiol 79:217-226

Paré D, Smith Y (1994) GABAergic projection from the intercalated cell masses of the amygdala to the basal forebrain in cats. J Comp Neurol 344:33-49.

Paré D, Smith Y, Paré JF (1995a) Intra-amygdaloid projections of the basolateral and basomedial nuclei in the cat: Phaseolus vulgaris-leucoagglutinin anterograde tracing at the light and electron microscopic level. Neuroscience 69:567-583.

Paré D, Pape HC, Dong J (1995b) Physiological properties of cat basolateral amygdaloid neurons: intracellular recordings in barbiturate-anesthetized cats. J Neurophysiol 74:1179-1191.

Pitkänen A, Amaral DG (1993) Distribution of parvalbumin-immunoreactive cells and fibers in the monkey temporal lobe: the hippocampal formation. J Comp Neurol 331:37-74.

Rainnie DG, Asprodini EK, Shinnick-Gallagher P (1991) Inhibitory transmission in the basolateral amygdala. J Neurophysiol 66:999-1009.

Rainnie DG, Asprodini EK, Shinnick-Gallagher P (1993) Intracellular recordings from morphologically identified neurons of the basolateral amygdala. J Neurophysiol 69:1350-1362.

Russchen FT (1986) Cortical and subcortical afferents of the amygdaloid complex. In: Excitatory amino acids and epilepsy (Schwarz R, Ben-Ari Y, eds), pp 35-52. New York: Plenum.
Savander V, Miettinen R, LeDoux JE, Pitkänen A (1997) Lateral nucleus of the rat amygdala is reciprocally connected with basal and accessory basal nuclei: a light and electron microscopic study. Neuroscience 77:767-781.

Smith Y, Paré D (1994) Intra-amygdaloid projections of the lateral nucleus in the cat: PHA-L anterograde labeling combined with post-embedding GABA and glutamate immunocytochemistry. J Comp Neurol 342:232-248.

Smith Y, Paré JF, Paré D (1998) Cat intraamygdaloid inhibitory network: ultrastructural organization of parvalbumin-immunoreactive elements. J Comp Neurol 391:164-179.

Smith Y, Paré JF, Paré D (2000) Differential innervation of parvalbuminimmunoreactive interneurons of the basolateral amygdaloid complex by cortical and intrinsic inputs. J Comp Neurol 416:496-508.

Sorvari H, Soininen H, Paljärvi L, Karkola K, Pitkänen A (1995) Distribution of parvalbumin-immunoreactive cells and fibers in the human amygdaloid complex. J Comp Neurol 360:185-212.

Stefanacci L, Farb CR, Pitkänen A, Go G, LeDoux JE, Amaral DG (1992) Projections from the lateral nucleus to the basal nucleus of the amygdala: a light and electron microscopic PHA-L study in the rat. J Comp Neurol 323:586-601.

Szinyei C, Heinbockel T, Montagne J, Pape HC (2000) Putative cortical and thalamic inputs elicit convergent excitation in a population of GABAergic interneurons of the lateral amygdala. J Neurosci 20:8909-8915.

Turner BH, Herkenham M (1991) Thalamoamygdaloid projections in the rat: a test of the amygdala's role in sensory processing. J Comp Neurol 313:295-325.

Washburn MS, Moises HC (1992a) Electrophysiological and morphological properties of rat basolateral amygdaloid neurons in vitro. J Neurosci 12:4066-4079.

Washburn MS, Moises HC (1992b) Inhibitory responses of rat basolateral amygdaloid neurons recorded in vitro. Neuroscience 50:811-830. 